\title{
General identifiability of possible world models for crowd wisdom
}

\author{
Drazen Prelec* and John McCoy ${ }^{\dagger}$
}

First version: April 25, 2020; this version: February 23, 2022

\begin{abstract}
Prelec, Seung and McCoy (2017) proposed a crowd wisdom model where ideal Bayesian observers receive discrete i.i.d. signals $s_{i} \in\left\{s_{1}, \ldots, s_{n}\right\}$ conditional on an unknown 'state,' i.e., a possible world $a_{j} \in\left\{a_{1}, \ldots, a_{m}\right\}$. Signals and worlds are presumed drawn from a distribution $p\left(s_{i}, a_{j}\right)$ known to observers, but unknown to the analyst. The paper asserted via an example but without formal proof that if ideal observers specify a belief matrix as conditional distributions $p\left(a_{j} \mid s_{i}\right)$, and a meta-knowledge matrix (beliefs about other observers' signals) as $p\left(s_{i} \mid s_{k}\right)=\sum_{j} p\left(s_{i} \mid a_{j}\right) p\left(a_{j} \mid s_{k}\right)$, then the analyst can, in the large sample limit, derive $p\left(s_{i}, a_{j}\right)$ and identify the actual signal-generating world. Here we provide a proof based on computing the signal prior as the stationary distribution of the meta-knowledge matrix.
\end{abstract}

\section{Background}

Here we provide a simple proof of the claim made in Prelec, Seung, and McCoy (2017), Supplements, Section 1.4:

It is possible to extend our approach to the general setting of $m$ worlds, and $n$ signals, provided one also elicits respondents' posterior distribution over possible answers.

${ }^{*}$ Sloan School of Management, Department of Economics, and Brain and Cognitive Sciences, E62-640, Massachusetts Institute of Technology; dprelec@mit.edu

${ }^{\dagger}$ The Wharton School, University of Pennsylvania; jpmccoy@ wharton.upenn.edu

${ }^{\ddagger}$ Lemma and Appendix are from 4/25/2020; revised for context and literature 1/25/2022. 
Prelec, Seung, and McCoy (2017) considered the 'crowd wisdom' problem of identifying the actual world in a possible world model, using data generated by an infinite sample of ideal observers (e.g., respondents to a survey) who report their 'signal' and possibly some further input. The 'actual world' might refer to the correct answer in a multiple choice question or a future event that experts attempt to predict. The 'signal' is a stochastic draw given the actual world. The 'model' is the joint distribution over signals and worlds. Observers know the model, but the analyst does not.

The traditional, and extensively researched, further input generated by observers is the posterior distribution over possible worlds, which quantifies the observer's beliefs as subjective probabilities. However, Theorem 1 in Prelec, Seung, and McCoy (2017) (Supplements) showed that such beliefs are always insufficient to identify the actual world: Any data consisting of $(a)$ the signal distribution, and $(b)$ the posteriors over worlds associated with each signal, can always be reconciled with any possible world being the actual world.

In the above excerpt from Prelec, Seung, and McCoy (2017), 'our approach' refers to methods where the further input is $(c)$ each observer's distribution over other respondents signals. For $m=2, n \geq 2$ and some special $n=m>2$ cases, inputs (a) \& (c) suffice to identify the actual world (Prelec, Seung, and McCoy (2017) Supplements, Sections 1.2, 1.3, Theorems 2 and 3) .

For the general case $n \geq 2, m \geq 2$, one requires both 'beliefs about worlds' and 'beliefs about signals,' as asserted in the excerpt above. Prelec, Seung, and McCoy (2017) provided an illustration of how this information can be used to find the actual world via a numerical example containing more worlds than signals, $m=3, n=2$, but no formal proof. Here we show how one can derive the possible world model from inputs (a) \& (b) \& (c), and thus identify the actual world. The main insight (noted in Prelec, Seung, and McCoy (2012), Box 3; or Prelec, Seung, and McCoy (2013), fn. 2), is that the matrix which contains distributions over signals can be viewed as a transition matrix for an irreducible discrete Markov chain, and theunique stationary distribution of this chainis the prior over signals. Because the conditional probabilities, worldgiven-signal, are presumed known, this pins down the model and allows the analyst to infer what distribution would appear in any possible world. Identifiability of the actual world is then guaranteed in the large sample limit. 


\section{Proof}

We first review the assumptions and notation in Prelec, Seung, and McCoy (2017). Possible answers to a question $a_{j}, j=1, \ldots, m$, are represented as possible worlds; the correct answer is the 'actual' world, $a_{i^{*}}$; the evidence available to each respondent is represented as a signal $s_{i}, i=1, \ldots, n$ generated i.i.d. from the actual world:

Respondents have two types of beliefs, both computed from their received signal $s_{i}$ and the joint distribution $p\left(s_{i}, a_{j}\right)$. Beliefs about the correct answer are given by the posterior probabilities $p\left(a_{j} \mid s_{i}\right)$, which can be obtained from knowledge of the joint distribution of signals and answers. Beliefs about signals received by other respondents, say the probability of another respondent receiving signal $s_{i}$ written as $p\left(s_{i} \mid s_{k}\right)$, are derived by computing the distribution of signals $p\left(s_{i} \mid a_{j}\right)$ conditional on a particular answer being correct, and marginalizing over all possible answers,

$$
p\left(s_{i} \mid s_{k}\right)=\sum_{j} p\left(s_{i} \mid a_{j}\right) p\left(a_{j} \mid s_{k}\right)
$$

More explicitly, one would write, $p\left(s_{i}^{q} \mid s_{k}^{r}\right)=\operatorname{Pr}\left(S^{q}=s_{i} \mid S^{r}=s_{k}\right)$, which is the probability that another, randomly selected respondent $q$ receives signal $s_{i}$ given that respondent $r$ has received signal $s_{k} \ldots$ (Prelec, Seung, and McCoy (2017), Supplements, 1.1)

Relative to the notation in Prelec, Seung, and McCoy (2017), we swap the column/row orientation for the signal matrix, so that worlds are consistently arranged as columns in the signal, belief, and possible world model matrices. Let:

- $Q: n \times m$ possible world model matrix where $Q_{i j}=p\left(s_{i}, a_{j}\right)>0$

- $S: n \times m$ signal matrix where $S_{i j}=p\left(s_{i} \mid a_{j}\right)$

- $B: n \times m$ belief matrix where $B_{i j}=p\left(a_{i} \mid s_{j}\right)$

- $M: n \times n$ meta-knowledge matrix where $M_{i j}=p\left(s_{i} \mid s_{j}\right)$

The positivity constraint on $Q_{i j}$ is crucial, ensuring that all signals are represented in the sample.

Lemma. Matrices $B$ and $M$ uniquely determine matrix $Q$. 
Proof. $M$ is evidently a stochastic matrix with positive entries, $\sum_{j} M_{i j}=1$ and $M_{i j}=\sum_{k} p\left(a_{k} \mid s_{j}\right) p\left(s_{i} \mid a_{k}\right) \geq$ $p\left(a_{i^{*}} \mid s_{j}\right) p\left(s_{i} \mid a_{i^{*}}\right)>0$. Interpreted as a transition matrix for a Markov chain, $M$ is irreducible and therefore has a unique stationary distribution, $\pi_{i}=\sum_{k} M_{i k} \pi_{k}$ (Parzen, 1999). This distribution is also the prior over signals, $\pi_{i}=p\left(s_{i}\right)$, as $p\left(s_{i}\right)=\sum_{k} p\left(s_{i}, s_{k}\right)=\sum_{k} p\left(s_{i} \mid s_{k}\right) p\left(s_{k}\right)$ for $i=1, \ldots, n$. The entries of $Q$ follow: $Q_{i j}=$ $p\left(s_{i}, a_{j}\right)=p\left(a_{j} \mid s_{i}\right) p\left(s_{i}\right)=B_{j i} \pi_{i}$.

The following assumption ensures that beliefs can differentiate among possible worlds.

Assumption. Stochastic relevance: If for all $s_{i}, p\left(a_{j} \mid s_{i}\right)=p\left(a_{k} \mid s_{i}\right)$, then $j=k$.

Proposition. Under stochastic relevance, the actual world $a_{i^{*}}$ is identifiable from $B, M$ and the signal distribution $p\left(s_{j} \mid a_{i^{*}}\right), j=1, \ldots, n$.

Proof. From the Lemma it follows that one can derive $Q$, and therefore $S$, from $B, M$. By the stochastic relevance assumption, the columns of $S$ are distinct. Therxxxxxefore, the actual signal distribution $p\left(s_{i} \mid a_{i^{*}}\right)$ will match only one column, which is the actual world.

Remark 1. The signal prior can be computed as the unit eigenvector of the meta-knowledge matrix (details in Appendix). Alternatively, one can use the following direct formula:

$$
p\left(s_{i}\right)=\left(\sum_{j} \frac{p\left(s_{j}\right)}{p\left(s_{i}\right)}\right)^{-1}=\left(\sum_{j} \frac{p\left(s_{j} \mid s_{i}\right)}{p\left(s_{i} \mid s_{j}\right)}\right)^{-1}=\left(\sum_{j} \frac{M_{j i}}{M_{i j}}\right)^{-1}
$$

The entries of the possible world model are then:

$$
p\left(s_{i}, a_{j}\right)=p\left(a_{j} \mid s_{i}\right) p\left(s_{i}\right)=B_{j i}\left(\sum_{j} \frac{M_{j i}}{M_{i j}}\right)^{-1}
$$

While either method will always yield a matrix $Q$, that $\mathrm{Q}$ may not regenerate the original matrix $M$ when eq. 1 is applied. That is, if the derived $Q$ is the correct possible world model, then the entries of the metaknowledge matrix should be: $M_{i j}=p\left(s_{i} \mid s_{j}\right)=\sum_{k} p\left(a_{k} \mid s_{j}\right) p\left(s_{i} \mid a_{k}\right)=\sum_{k} \frac{q_{j k}}{\sum_{l} q_{l k}} \frac{q_{i k}}{\sum_{l} q_{i l}}$. But there is no guarantee that: $\sum_{k} \frac{q_{j k}}{\sum_{l} q_{l k}} \frac{q_{i k}}{\sum_{l} q_{i l}}=M_{i j}$, if $q_{i j}=B_{j i} \pi_{i}$ and $\pi_{i}$ is the stationary distribution of $M$. The method therefore assumes that matrices $B$ and $M$ correspond exactly to $Q$, i.e., there is no error in reporting $B$ and $M$. 
Remark 2. It is straightforward to construct incentives for honest reporting of all three inputs even when the correct answer is not known to the analyst. Honest answers could be incentivized with mechanisms such as the Bayesian truth serum, which also includes incentives for honest predictions of the signal distribution (Prelec, 2004). Because the algorithm identifies the actual world in the large sample limit, beliefs may be incentivized with any proper scoring rule, e.g., the logarithmic scoring rule (Savage, 1971).

\section{Finite case}

A finite sample of ideal observers will specify $B, M$ if at least one observer reports each signal. One can then generate a Bayesian posterior distribution over worlds by applying Bayes' rule. The finite case provides some additional insight into how the method works. Let $C_{i}$ denote the count of reported signals $i$, and $C=\sum_{i} C_{i}$ the total sample size. Because signals are i.i.d.,

$$
\begin{aligned}
\operatorname{Pr}\left(a_{j} \mid C_{1}, \ldots, C_{n}\right) & =\frac{\operatorname{Pr}\left(C_{1}, \ldots, C_{n} \mid a_{j}\right)}{\operatorname{Pr}\left(C_{1}, \ldots, C_{n}\right)} p\left(a_{j}\right) \\
& \propto \operatorname{Pr}\left(C_{1}, \ldots, C_{n} \mid a_{j}\right) p\left(a_{j}\right) \\
& =p\left(s_{1} \mid a_{j}\right)^{C_{1}} \times \cdots \times p\left(s_{n} \mid a_{j}\right)^{C_{n}} p\left(a_{j}\right) \\
& =\left(\frac{p\left(a_{j} \mid s_{1}\right) p\left(s_{1}\right)}{p\left(a_{j}\right)}\right)^{C_{1}} \times \cdots \times\left(\frac{p\left(a_{j} \mid s_{n}\right) p\left(s_{n}\right)}{p\left(a_{j}\right)}\right)^{C_{n}} p\left(a_{j}\right) \\
& =p\left(a_{j} \mid s_{1}\right)^{C_{1}} \times \cdots \times p\left(a_{j} \mid s_{n}\right)^{C_{n}} p\left(a_{j}\right)^{1-C} \prod_{k} p\left(s_{k}\right) \\
& \propto p\left(a_{j} \mid s_{1}\right)^{C_{1}} \times \cdots \times p\left(a_{j} \mid s_{n}\right)^{C_{n}} p\left(a_{j}\right)^{1-C}
\end{aligned}
$$

This is a standard expression, normally applied when the analyst has a prior over worlds i.e., 'hypotheses' $a_{j}$. With our method, the prior is generated by the crowd, $p\left(a_{j}\right)=\sum_{k} \pi_{k} B_{j k}$, yielding:

$$
\operatorname{Pr}\left(a_{j} \mid C_{1}, \ldots, C_{n}\right) \propto B_{j 1}^{C_{1}} \times \cdots \times B_{j n}^{C_{n}}\left(\sum_{k} \pi_{k} B_{j k}\right)^{1-C}
$$


Let $\bar{x}_{j}=\lim _{C \rightarrow \infty} C_{j} / C$, the asymptotic relative frequency of signal $s_{j}$. Using eq. 3 , we can infer the actual world that is the source of signals in the large sample limit:

$$
\begin{aligned}
a_{i^{*}} & =\underset{a_{j}}{\arg \max }\left\{\lim _{C \rightarrow \infty} \operatorname{Pr}\left(a_{j} \mid C_{1}, \ldots, C_{n}\right)\right\} \\
& =\underset{a_{j}}{\arg \max }\left\{\lim _{C \rightarrow \infty} B_{j 1}^{C_{1}} \times \cdots \times B_{j n}^{C_{n}}\left(\sum_{k} \pi_{k} B_{j k}\right)^{1-C}\right\} \\
& =\underset{a_{j}}{\arg \max }\left\{\lim _{C \rightarrow \infty}\left(B_{j 1}^{C_{1}} \times \cdots \times B_{j n}^{C_{n}}\left(\sum_{k} \pi_{k} B_{j k}\right)^{1-C}\right)^{1 / C}\right\} \\
& =\underset{a_{j}}{\arg \max }\left\{B_{j 1}^{\bar{x}_{1}} \times \cdots \times B_{j n}^{\bar{x}_{n}}\left(\sum_{k} \pi_{k} B_{j k}\right)^{-1}\right\}
\end{aligned}
$$

There are two finite cases of special interest. With a uniform prior over worlds, the posterior probability is proportional to the product of reported probabilities, which is the well known 'logarithmic pool' (Cooke, 1991):

$$
\operatorname{Pr}\left(a_{j} \mid C_{1}, \ldots, C_{n}\right) \propto B_{j 1}^{C_{1}} \times \cdots \times B_{j n}^{C_{n}}
$$

With a uniform prior over signals rather than worlds, we obtain a less familiar 'democratic pool:'

$$
\operatorname{Pr}\left(a_{j} \mid C_{1}, \ldots, C_{n}\right) \propto B_{j 1}^{C_{1}} \times \cdots \times B_{j n}^{C_{n}} \bar{B}_{j}^{1-C}
$$

where $\bar{B}_{j}$ is the prior on world $a_{j}$ computed as a simple average, not weighted by counts: $p\left(a_{j}\right)=\bar{B}_{j}=$ $m^{-1} \sum_{k} B_{j k}$. The label 'democratic' reflects an interpretation of signals as referring to people of the given class or type, and hence having the same beliefs about the world. In eq. 4, the weight of opinions of people with a given type is ex-ante unbiased; the aggregated probability only reflects their relative frequency in the sample, which is also the key feature of democratic voting. Formally, eq. 4 obtains if $M$ is doubly-stochastic, both rows and columns summing to one (Prelec, Seung, and McCoy, 2012).

Remark 3. In empirical work with noisy data we have found that such posterior probabilities are not well calibrated for large samples, and should therefore be interpreted with caution. That is, if $B, M$ are estimated by averaging respondents' inputs, and the average interpreted as exact (without error), then probabilities will quickly converge to either zero or one as the sample size increases, i.e., they will be overconfident. To produce reasonable probabilistic estimates with large samples, one needs to introduce a noise model for $B, M$ reports (McCoy and Prelec, 2017). 
Remark 4. Eq. 3, provides a tie-breaking criterion when there are two observers that disagree on a binary proposition, with $B_{11}>0.5, B_{22}>0.5$ (Koriat, 2012; Bahrami et al., 2010). Normally, one would rely on relative confidence to decide which observer is more likely to be correct. For example, if $B_{11}>B_{22}$, the analyst would conclude that observer 1 is more confident, and therefore world 1 more likely. Our model additionally takes into account prior probabilities of signals and worlds. Using the expression for the 2-signal, 2-world case, we have:

$$
\frac{\operatorname{Pr}\left(a_{1} \mid 1,1\right)}{\operatorname{Pr}\left(a_{2} \mid 1,1\right)}=\frac{p\left(a_{1} \mid s_{1}\right) p\left(a_{1} \mid s_{2}\right) p\left(a_{1}\right)^{-1}}{p\left(a_{2} \mid s_{1}\right) p\left(a_{2} \mid s_{2}\right) p\left(a_{2}\right)^{-1}}=\frac{B_{11}\left(1-B_{22}\right) p\left(a_{1}\right)^{-1}}{\left(1-B_{11}\right) B_{22} p\left(a_{2}\right)^{-1}}
$$

If there are two signals, then the prior over signals is (eq. 2):

$$
\pi_{1}=\frac{M_{12}}{M_{12}+M_{21}}, \pi_{2}=\frac{M_{21}}{M_{12}+M_{21}}
$$

and the prior over worlds:

$$
p\left(a_{1}\right)=\pi_{1} B_{11}+\pi_{2} B_{12}, p\left(a_{2}\right)=\pi_{1} B_{21}+\pi_{2} B_{22}
$$

World 1 is more likely if $\operatorname{Pr}\left(a_{1} \mid 1,1\right)>\operatorname{Pr}\left(a_{2} \mid 1,1\right)$, or:

$$
\frac{B_{11}\left(1-B_{22}\right)}{\left(1-B_{11}\right) B_{22}}>\frac{p\left(a_{1}\right)}{p\left(a_{2}\right)}=\frac{M_{12} B_{11}+M_{21}\left(1-B_{22}\right)}{M_{21} B_{22}+M_{12}\left(1-B_{11}\right)}
$$

Relative confidence (left side) is compared against prior world odds (right side). Put another way, a confidence level for a world that is a priori unlikely has more weight than the same confidence level for the world that is a priori expected.

\section{Related work}

The idea of computing a prior as a stationary distribution of a Markov transition matrix was introduced by D. Samet, in a multi-player context (Samet, 1998). More directly relevant to our problem is the recent regression approach introduced by J. Libgober (Libgober, 2021). The theoretical setup is different insofar as a single 
agent is tasked with specifying the complete matrices $B$ and $M$. For each signal $s_{i}$, one can then estimate the $m$ coefficients $S_{i k}, k=1, \ldots, m$, as parameters in a regression of a 'y-variable' $n$-vector $M_{i j}, j=1, \ldots, n$, against the 'x-variables' matrix $B_{k j}, j=1, \ldots, m$ (as $M_{i j}=\sum_{k} S_{i k} B_{k j}$, by definition, eq. 1). If $n \geq m$, then simple OLS will produce the desired row of $S$-values. If $n<m$, one can resort to ridge regression (details in Libgober 2021). The priors over worlds or signals can then be computed by the same stationary distribution method as discussed here.

Under our model assumptions all methods will yield the same true possible world model $Q$. However, with an arbitrary pair of matrices $B, M$ (i.e., a pair not constructed from some $Q$ ) the different estimation methods will generally yield different results. An advantage of the linear regression approach is that it can be applied even if some signals are not sampled; that is, the linear regression method does not require a square matrix $M$. In the context of our problem, this suggests that the positivity requirement on $Q$ can in principle be removed. A conceptual drawback of linear regression is it may yield probabilities that are negative or greater than one. In contrast, the approach that begins by deriving the signal prior will always produce a proper possible world model $Q$. This opens the door to theoretical interpretations of discrepancies between the observed matrices $B, M$ and the matrices that would be consistent with the derived $Q$. For example, one could rationalize such discrepancies with a expanded model in which observers with different signals have different priors over signals, and thus also different possible world models.

\section{References}

Bahrami, Bahador et al. (2010). “Optimally interacting minds”. Science 329.5995, pp. 1081-1085.

Cooke, R.M. (1991). Experts in uncertainty: opinion and subjective probability in science. Oxford University Press, USA.

Koriat, A. (2012). "When Are Two Heads Better than One and Why?" Science 336.6079, pp. 360-362.

Libgober, Jonathan (2021). “Hypothetical Beliefs Identify Information”. arXiv:2105.07097.

McCoy, John and Dražen Prelec (2017). “A statistical model for aggregating judgments by incorporating peer predictions". arXiv:1703.04778.

Parzen, Emanuel (1999). Stochastic processes. SIAM.

Prelec, Dražen (2004). “A Bayesian truth serum for subjective data”. Science 306.5695, pp. 462-466. 
Prelec, Dražen, H Sebastian Seung, and John McCoy (2012). "Finding truth even if the crowd is wrong". MIT Working Paper.

— (2013). "Finding truth even if the crowd is wrong". MIT Working Paper.

— (2017). "A solution to the single-question crowd wisdom problem”. Nature 541.7638, pp. 532-535.

Samet, Dov (1998). "Iterated expectations and common priors". Games and economic Behavior 24.1-2, pp. $131-141$.

Savage, Leonard J (1971). "Elicitation of personal probabilities and expectations". Journal of the American Statistical Association 66.336, pp. 783-801.

\section{Appendix}

For completeness, and to facilitate algorithmic coding, we provide an explicit matrix equation that derives $Q$ from $B$ and $M$. Let:

$P: n \times n$ diagonal matrix, with signal prior on the diagonal, $P_{i i}=p\left(s_{i}\right), P_{i k}=0, i \neq k$

I: $n \times n$ identify matrix

$A^{T}$ : transpose of any matrix $A$

$A^{+}$: matrix derived from matrix $A$ by adding a column $(1, \ldots, 1)^{T}$ to the right

$b^{n+1}:(n+1)$ column vector composed of $n$ zeros, followed by single $1,(0, \ldots, 0,1)^{T}$

$e_{i}^{n}: n$ row vector, with zeros everywhere except at $i$-th entry,

$$
e_{i}^{n}(k)= \begin{cases}1 & \text { if } i=k \\ 0 & \text { otherwise }\end{cases}
$$

$E_{i}^{n}$ : diagonal $n \times n$ matrix with zeros everywhere except for the $i$-th diagonal entry,

$$
E_{i}^{n}(j, k)= \begin{cases}1 & \text { if } i=j=k \\ 0 & \text { otherwise }\end{cases}
$$

We first use the standard formula for the stationary distribution of Markov matrix $M$ :

$$
\left(p\left(s_{1}\right), \ldots, p\left(s_{n}\right)\right)^{T}=\left((M-I)^{+} \times\left((M-I)^{+}\right)^{T}\right)^{-1} \times(M-I)^{+} \times b^{n+1}
$$


to compute the signal prior. We then convert the column prior $\left(p\left(s_{1}\right), \ldots, p\left(s_{n}\right)\right)^{T}$ into a diagonal matrix $P$ with prior entries on the diagonal:

$$
P \equiv \sum_{i=1}^{n} E_{i}^{n} \times\left(p\left(s_{1}\right), \ldots, p\left(s_{n}\right)\right)^{T} \times e_{i}^{n}
$$

and multiply the diagonalized prior by the belief matrix to obtain the PWM model:

$$
Q=P \times B
$$

Coded as a single line from inputs $B$ and $M$ :

$$
Q=\left(\sum_{i=1}^{n} E_{i}^{n} \times\left(\left((M-I)^{+} \times\left((M-I)^{+}\right)^{T}\right)^{-1} \times(M-I)^{+} \times b^{n+1}\right) \times e_{i}^{n}\right) \times B
$$

